\title{
EL GRAN IMPULSO Y EL CRECIMIENTO BALANCEADO Y DESBALANCEADO*
}

Lauchlin Currie

"DOI: https://doi.org/10.18601/01245996.v20n39.04. Este es el capítulo 19 de un libro inédito sobre desarrollo económico que Lauchlin Currie (1902-93) escribió en 1969-1971 en la Universidad Simon Fraser, Canadá. Se publica con el amable permiso de su hija Elizabeth Currie. Traducción de Alberto Supelano y Luis Lorente. Recepción: 09-01-2018, aceptación: 11-05-2018. Sugerencia de citación: Currie, L. (2018). El Gran Impulso y el crecimiento balanceado y desbalanceado. Revista de Economía Institucional, 20(39), 69-92. 


\section{El Gran Impulso y el crecimiento balanceado y desbalanceado}

Resumen. Este artículo, que es a la vez una contribución a la historia del pensamiento económico y una aclaración de la teoría del desarrollo económico en la que se basó el Plan de Desarrollo colombiano de 1970-1974, Las cuatro estrategias, discute la propuesta de Paul Rosenstein-Rodan para acelerar el crecimiento en los países en desarrollo, y critica los puntos de vista de Albert Hirschman sobre el falso problema crecimiento balanceado-crecimiento desbalanceado que, en opinión del autor, dilapidó mucho tiempo y muchos esfuerzos, y, desvió la atención de los problemas verdaderos que debían ser tema de la teoría del desarrollo y de las políticas para acelerar el crecimiento económico y salir del subdesarrollo.

Palabras clave: Rosenstein-Rodan, Gran Impulso, crecimiento balanceado, crecimiento desbalanceado, Albert Hirschman, eslabonamientos; JEL: B10, D31, D51.

\section{The Big Push and Balanced and Unbalanced Growth}

Abstract. This article, which is both a contribution to the history of economic thought and a clarification of the theory of economic development on which the Colombian Development Plan of 1970-1974 was based, The Four Strategies, discusses the proposal of Paul Rosenstein -Rodan to accelerate growth in developing countries, and criticizes Albert Hirschman's views on the false problem of balanced growthunbalanced growth that, in the author's opinion, wasted a lot of time and effort, and diverted attention from the real problems that should be the subject of development theory and policies to accelerate economic growth and exit from underdevelopment.

Keywords: Rosenstein-Rodan, Big Push, balanced growth, unbalanced growth, Albert Hirschman, linkages; JEL: B10, D31, D51.

\section{O Grande Impulso e o crescimento balanceado e desbalanceado}

Resumo. Este artigo, além de contribuir para a história do pensamento econômico ao mesmo tempo que esclarece a teoria do desenvolvimento econômico na qual o Plano de Desenvolvimento colombiano de 1970-1974 se baseou, as quatro estratégias, discute a proposta de Paul Rosenstein-Rodan para acelerar o crescimento nos países em desenvolvimento e critica os pontos de vista de Albert Hirschman sobre o falso problema do crescimento balanceado-crescimento desbalanceado que, na opinião do autor, gastou muito tempo e muitos esforços, além de desviar a atenção dos reais problemas que deviam ser tema da teoria do desenvolvimento e das políticas para acelerar o crescimento econômico e sair do subdesenvolvimento.

Palavras-chaves: Rosenstein-Rodan, Grande Impulso, crescimento balanceado, crescimento desbalanceado, Albert Hirschman, encadeamentos; JEL: B10, D31, D51. 
T a necesidad de un esfuerzo masivo para activar o acelerar el proLceso de crecimiento, lograr el despegue, escapar del círculo vicioso de la pobreza o de la trampa del subdesarrollo, hacer el esfuerzo crítico mínimo para un crecimiento sostenido, dar un Gran Impulso, lograr el rompimiento o la transición a la categoría de país desarrollado, y los medios para hacer todo esto, que deberían ser los principales problemas tratados por la literatura y la política del subdesarrollo, nunca han recibido un análisis adecuado.

Al menos parte de la explicación se puede encontrar en que se ha creado un falso problema -el del cambio balanceado frente al desbalanceado- al cual se ha supeditado el problema real que subyace al Gran Impulso, o bien en el error de identificar la propuesta del Gran Impulso con alguna teoría particular del crecimiento.

De ahí que la necesidad del esfuerzo crítico o mínimo, así como los requisitos para su efectividad, nunca recibieran la atención que ameritaba su importancia. Por esta razón, examinaremos aquí la disyuntiva del crecimiento balanceado frente al desbalanceado, pues el problema real que esta discusión oculta parece estar desapareciendo de la literatura (aunque no de las recopilaciones de lecturas y de los requisitos para examen de posgrado).

Es una historia muy curiosa, pues en retrospectiva no parece que hubiera un problema real entre balance y desbalance. Al menos, ningún autor destacado elaboró una teoría del crecimiento balanceado en el sentido de explicar el crecimiento en términos de balance ${ }^{1}$, salvo que se pueda decir que la idea de balance está implícita y es inherente al funcionamiento de la economía de libre empresa y al mecanismo de mercado de fijación de precios.

En el fondo, los conceptos de balance, equilibrio y eficiencia son indistinguibles: describen la asignación de factores en respuesta o anticipación a la demanda, y la relación entre los costos marginales, de modo que exista una tendencia a la igualación de las rentabilidades. Los conceptos de producto máximo y de bienestar social reflejan un objetivo de equilibrio o balance (aunque nunca se alcance). Como dijo Scitovsky, la presencia de ganancias es en sí misma una indicación de desequilibrio; en un equilibrio perfecto, las ganancias desaparecerían. Pero, casi sobra decirlo, este es solo un estado que nadie espera ver realizado. En suma, así como el equilibrio implica una asignación o

1 Nurkse (1957) estuvo a punto de hacerlo en sus conferencias de 1957 en Estambul, que fueron relativamente desconocidas, pero como argumentaré no en su muy conocido Problemas de formación de capital en países subdesarrollados (1953) al que se dirigieron los ataques. 
"balance" de los esfuerzos productivos de acuerdo con la demanda, que iguala los costos marginales con las utilidades marginales, la realidad implica un desequilibrio o desbalance puesto que jamás alcanzamos ese equilibrio.

Así, una "teoría” del crecimiento balanceado que pretenda explicar el proceso real de crecimiento se limitaría a decir que la maximización de beneficios, los incentivos para mejorar la condición propia o "tener éxito", las condiciones de movilidad, de competencia y de operación del mecanismo de fijación de precios llevarían al desarrollo o al crecimiento, en un entorno físico y social adecuado.

En realidad, esto parece ser lo que sucedió en el caso de los países desarrollados no socialistas en las primeras etapas de su historia. Los intentos de explicar su desarrollo en términos de intervención del Estado -de fuerzas ajenas al mercado- no son convincentes, excepto quizá en el caso de Japón. Pero incluso en este caso, el asunto se trató como un caso en que el Estado interviene, pero no para crear desequilibrio o desbalance sino para que el entorno sea más favorable para el funcionamiento de las fuerzas del mercado. E1 otro tipo de intervención estatal -la que busca corregir deficiencias en la distribución y proporcionar servicios que no proporciona adecuadamente el mercado- podría explicarse y defenderse por las mismas razones, pero nunca como una estrategia para crear "desbalances" que obliguen a "tomar decisiones".

En general, la "doctrina" o "teoría” del crecimiento balanceado se atribuye a tres autores: Rosenstein-Rodan, Scitovsky y Nurkse. Es interesante volver a las fuentes originales y considerar lo que estos autores dijeron realmente.

El artículo pertinente de Rosenstein-Rodan se publicó en 1943 con el título de "Problemas de industrialización de Europa Oriental y Suroriental". Su tema principal era una propuesta para acelerar la industrialización de Europa Suroriental después de la guerra, mediante un programa masivo de ayuda extranjera que empleara los superabundantes recursos de trabajo, ocultos en el alto desempleo agrícola disfrazado, mediante una expansión ampliamente diversificada de industrias capaces de generar empleos y pagar salarios. No se presentó como una "teoría del crecimiento", sino como una propuesta específica para enfrentar lo que el autor consideraba una situación de emergencia (y lo era), semejante al Plan Marshall que se propuso y adoptó pocos años después para acelerar la reconstrucción de Europa Occidental.

Pero, para respaldarla, Rosenstein-Rodan usó dos argumentos que sus críticos aprovecharon. Uno mencionaba las economías externas 
que gestaría un programa de gasto a gran escala, y es indudable que un aumento del tamaño del mercado generaría algunas economías de escala internas y externas, como argumentó antes Allyn Young (1928), pero esto no era realmente necesario para el planteamiento de Rosenstein-Rodan. Bastaba demostrar que una expansión general del mercado justificaría e induciría muchas inversiones que, de otro modo, no se harían. El problema consistía en asegurar que la demanda de los consumidores aumentara lo suficiente para proporcionar un mercado para el producto de las nuevas industrias de bienes de consumo que él proponía, de manera que hubiese un mercado permanente para los bienes que producirían en el futuro los subempleados del presente.

Para este propósito, debería haberse interesado más por discutir las consecuencias de la inelasticidad de la demanda en bienes de consumo general y en la necesidad de compensar las continuas fugas de recursos por el crecimiento de los ahorros, en vez de preocuparse por la eventual disminución de los costos unitarios que pudiera lograr. Es como si Keynes hubiese intentado justificar su propuesta de expansión de la demanda agregada en medio de la Gran Depresión mencionando las economías de escala que generaría dicha expansión, en vez de concentrarse en el crecimiento directo del producto y el empleo que resultaría del mayor gasto. Aunque válida, la introducción de las economías externas en el argumento tendía a desviar la atención del tema principal.

El concepto de balance estaba implícito en la idea de que "las industrias que producen la mayor parte de los bienes salario se pueden considerar complementarias" (p. 206) en el sentido de que cada una genera una demanda para los productos de las demás, lo que, a su vez, sugiere que la asignación de los fondos de inversión debía prestar atención a las elasticidades ingreso de la demanda mencionadas y a sus posibles cambios, sugerencia razonable si se emprendiera tal programa de inversión. No menciona explícitamente el obstáculo de la inelasticidad de demanda de los bienes de consumo masivo, pero está claramente implícito en el muy citado ejemplo de la fábrica de calzado y en la idea de complementariedad que, a su vez, se basa en la observación de que la elasticidad ingreso de la demanda para el conjunto de todos los bienes de consumo es uno. (Él era consciente de las fugas keynesianas, pero no sugirió cómo se podían compensar; un defecto de su propuesta fácilmente remediable.) Más adelante, Nurkse introdujo con mayor claridad el concepto de inelasticidad de la demanda de un bien, pero no logró entender sus implicaciones de 
largo alcance para la naturaleza y la rapidez del proceso de crecimiento y para los fines de la política.

Cabe imaginar que Rosenstein-Rodan se sorprendió al enterarse, más tarde, que su audaz sugerencia de intervención del Estado para lograr rápidamente lo que los mecanismos del mercado solo lograrían lentamente, si lo lograban, se había transformado en el enunciado de una teoría o doctrina del crecimiento; y no solo del crecimiento sino del crecimiento o desarrollo balanceado. No era más que una propuesta práctica de política y habría sido igualmente justo describirla como una sugerencia de crecimiento desbalanceado: la expansión deliberada del sector de bienes de consumo no agrícolas para corregir el desbalance causado por el excesivo número de personas en el sector agrícola y por una escasez de fuentes externas de capital. La expansión anticipada del mercado crearía, a su vez, "desequilibrio, tensiones y posibilidades de ganancias" y tendría “eslabonamientos horizontales y verticales", todo lo cual actuaría para inducir nuevas inversiones, mayor absorción de las fuerzas agrícolas redundantes y crecimiento de ciertos sectores con respecto a otros. En otras palabras, sería tan razonable decir que Rosenstein-Rodan defendía el crecimiento desbalanceado como decir que era un exponente del crecimiento balanceado. Pero ninguna de estas caracterizaciones sería adecuada porque, lo que realmente estaba en juego, era la conveniencia, en las circunstancias prevalecientes, de un esfuerzo deliberadamente grande para acelerar la industrialización.

Con más justicia, se puede atribuir a Ragnar Nurkse (1953) una preocupación más general y un análisis más profundo del crecimiento balanceado, aunque tampoco era esta su tesis central. No la discutió como una teoría del crecimiento, sino como un análisis del funcionamiento y de las fallas de la Ley de Say y del mecanismo de fijación de precios. De nuevo, en la afirmación "la dificultad se desvanece, al menos en principio, en el caso de una aplicación más o menos sincronizada del capital a una amplia gama de industrias diferentes" (ibíd., p. 11), la idea de balance en el sentido de ampliar el producto $y$, por tanto, la inversión de acuerdo con una diversidad de demandas efectivas, aparecía como una estrategia para escapar de la trampa del subdesarrollo que surge al considerar cada bien de consumo por separado y constatar la inelasticidad de su demanda. A menos que haya errores de criterio en las inversiones, cuanto más amplio y diverso sea el aumento de la producción, mayor será la demanda de productos para todas y cada una de las empresas y mejor funcionará la Ley de Say. Aquí es donde entra la idea de "balance", es decir, de un balance 
entre la producción adicional y las elasticidades ingreso de la demanda en condiciones de producción general rentable.

Es importante señalar que, en su libro de 1953, Nurkse no hizo mucho énfasis en la cuestión del balance. Habló de "aplicación más o menos sincronizada" y mencionó con aprobación el concepto de oleadas o racimos de innovaciones e inversiones de Schumpeter, pero no se sintió perturbado en lo más mínimo por la idea de un crecimiento que oscilara por encima y por debajo de una línea de tendencia. Si se suscribió a una teoría particular del crecimiento, fue a la de Schumpeter. Pero también estaba dispuesto a aceptar la conveniencia de intervenciones del gobierno para promover el crecimiento. "Puede ser que [...] las fuerzas que venzan la parálisis del estancamiento económico deban organizarse deliberadamente en alguna medida, al menos inicialmente" (ibíd., p. 15), y mencionó con beneplácito la temprana intervención económica del gobierno japonés. Advirtió que tal intervención puede dar lugar a errores - “desbalances"- pero continuó diciendo que "las desproporciones de un tipo u otro también son una característica de los auges cíclicos a través de los cuales la empresa privada logró el progreso económico" (ibíd.,p. 16). E1 hecho es que no estaba realmente comprometido con los métodos usados para acelerar el desarrollo, ni con una teoría o explicación del desarrollo, sino que solo insistía en el papel clave o central de la formación de capital, o de su ausencia, para el proceso. De hecho, fue esta insistencia, que consideré excesiva, lo que me llevó a pensar que la influencia de su trabajo no era tan constructiva como podría haber sido, y a descuidar los pasajes constructivos sobre las posibilidades y la conveniencia de los métodos a gran escala, pero "más o menos" sincronizados, para ampliar el mercado, la producción y el empleo mencionados anteriormente ${ }^{2}$.

$\mathrm{E} 1$ tercer autor al que se suele citar como defensor de la teoría del balance es Scitovsky (1954). En su conocido artículo sobre economías externas repitió las observaciones de Rosenstein-Rodan y de Nurkse sobre los problemas suscitados por un mercado pequeño. Extendió el concepto de economías externas para cubrir todos los beneficios que puede traer el mayor tamaño de un mercado, señalando que los cálculos de la rentabilidad privada subestiman la conveniencia social cuando hay economías externas, porque el cálculo privado no toma en cuenta el impacto que tendrán sus decisiones para hacer más rentables las operaciones de otras empresas. Después, al examinar el modelo de dos industrias, concluyó que las limitaciones a la inversión provenien-

${ }^{2}$ Ver también mi discusión de las opiniones de Nurkse en Currie (1966, cap. 9). 
tes de la estrechez del mercado "solo pueden eliminarse totalmente mediante la expansión simultánea de ambas industrias”. Apoyó así la política del Gran Impulso, afirmando que "las ganancias en una economía mixta son una mala guía del óptimo económico en lo que respecta a la inversión y la expansión industrial, y son peores cuanto más descentralizada y diferenciada es la economía" (ibíd., p. 149).

$¿$ Es este, entonces, un argumento en favor de un crecimiento balanceado? De nuevo, es una cuestión de semántica. Scitovsky (1954) identificó el balance con el equilibrio y el equilibrio como la antítesis del crecimiento. Más tarde, Scitovsky (1959) escribió un artículo en el que expuso en detalle este punto de vista. En su artículo anterior afirmó que el análisis de una teoría de equilibrio es aplicable cuando no hay ganancias. A la implementación de la expansión planeada le prestó aún menos atención que RosensteinRodan. Para situarlo en el campo del crecimiento balanceado, habría que poner un énfasis indebido en su uso casual de las palabras "simultáneo" e "integrado". En suma, su interés y su preocupación eran extender el concepto de economías externas y no elaborar una "teoría" del crecimiento, balanceado o no. Pero creía que el concepto de economías pecuniarias externas justificaba una expansión planeada de la inversión en una economía subdesarrollada, la cual sería una politica de desarrollo.

Es interesante observar que ninguno de los tres autores a los que se suele atribuir la defensa o elaboración de una "teoría del crecimiento balanceado" se considerara como tal. Todos estaban interesados, en mayor o menor grado, en el problema de acelerar el desarrollo (o preocupados por las bajas tasas de crecimiento observadas) y subrayaban que un pequeño mercado inicial constituía un obstáculo para inducir la inversión. Todos creían que el éxito genera éxito o que el crecimiento genera crecimiento, que cuanto más amplia sea la base del movimiento de desarrollo más probable es que genere demanda, empleo, ganancias, inversión y mayor crecimiento. Pensaban que la inversión planeada, bien sea privada o pública, debería estar calibrada por la demanda que se espera inducir, para que sea posible minimizar las pérdidas o la capacidad no utilizada. Este último rasgo es el que se quiso interpretar como la característica esencial de la propuesta y al que se etiquetó “crecimiento balanceado". Pero en realidad Rosenstein-Rodan estaba interesado principalmente en acelerar la industrialización de posguerra y emplear el exceso de trabajo agrícola, Nurkse en las formas y medios de estimular la demanda y la oferta de capital, y Scitovsky en aclarar los conceptos de economías externas. 
Marcus Fleming, en su artículo de 1955 titulado "Las economías externas y la doctrina del crecimiento balanceado", señaló que "el argumento de que proyectos de inversión que no son rentables individualmente pueden serlo cuando se adoptan en conjunto, y por tanto son una buena cosa", se suele denominar doctrina del crecimiento balanceado. De modo que, para esa fecha, un rasgo de la propuesta de Rosenstein-Rodan, que aparece en el análisis de la trampa del subdesarrollo y del incentivo para invertir de Nurkse (1953) y en el análisis de las economías externas de Scitovsky (1954), se identificó en la literatura como la doctrina del crecimiento balanceado. Así, los méritos de la propuesta original quedaron ligados a un detalle del análisis del círculo de la pobreza de Nurkse.

Una cosa extraña en este extraño episodio de la historia del pensamiento económico es que Nurkse realmente no parecía estar muy interesado en la propuesta de Rosenstein-Rodan y, en su exposición de la trampa del subdesarrollo, adoptó supuestos contrarios a los de este último. Por ejemplo, para la validez de la propuesta de Rosenstein-Rodan era fundamental que hubiese una oferta prácticamente ilimitada de trabajo "excedente". En cambio, para su argumento en favor de una gama de inversiones muy diversificada, Nurkse supuso, como hizo Fleming, una oferta elástica de capital y una oferta fija de trabajo.

En 1957, sin embargo, Nurkse (1957) había aceptado la designación de "principio", "doctrina" o "noción" del crecimiento balanceado, pero veía el problema más en términos de especialización internacional que de desarrollo industrial o agrícola interno de los países subdesarrollados. Prestó poca atención al Gran Impulso en Los problemas de la formación de capital (1954) y ninguna en absoluto en el artículo de 1957.

En suma, en el capítulo que recibió tanta atención, Nurkse nunca estableció el "balance" como principio o doctrina del crecimiento, sino como factor o condición limitante. Lo dijo expresamente: "E1 crecimiento balanceado puede ser algo bueno en sí mismo, pero aquí nos interesa principalmente por sus efectos sobre la demanda de capital" (p. 12). Como economista, habría preferido sin duda que las inversiones se utilizaran plenamente y fueran rentables. Pero la principal preocupación de su libro era aumentar el producto de los países subdesarrollados aumentando la formación de capital. El balance no era algo que se buscara por sí mismo, sino solo como condición que favorecería el crecimiento, acelerándolo mediante incentivos para invertir y el éxito de la inversión. 
Es una lástima que Nurkse no tratara más a fondo el papel de los incentivos para producir y su importancia para el problema del "equilibrio del subdesarrollo" (p. 10), en vez limitarse a discutir las condiciones de la oferta de capital, pues terminó subrayando la importancia de la formación de capital para el crecimiento, pero sin plantear una política positiva que indujera más inversiones y descuidando otros factores del proceso de crecimiento. Sea o no válida la historia de las oleadas de innovación de Schumpeter, difícilmente serían la base de una politica de desarrollo consciente y sistemática, y esto es evidentemente lo que se necesita, salvo, por supuesto, que se piense que bastan las fuerzas del mercado para que la población en rápido crecimiento de los países subdesarrollados aproveche al máximo la tecnología moderna.

Es tentador revisar la bibliografía posterior a la que contribuyeron muchos autores distinguidos, incluidos Fleming, Singer, Streeten, Sheahan, Scitovsky, Montias, Lipton y Mathur, entre otros, pero esto me alejaría demasiado del argumento principal de este trabajo. $\mathrm{Me}$ limitaré, por tanto, a discutir el tratamiento de Hirschman porque pienso que gran parte de la desafortunada confusión se deriva de su trabajo, que fue ampliamente citado, y que él intentó convertir en una teoría del crecimiento y en fundamento de una política.

En su tratamiento de los problemas (Hirschman, 1958) logró confundir todo identificando el Gran Impulso con la discusión del balance, y los juntó como una "teoría del desarrollo", lo que con toda seguridad nunca fueron. Después de crear algo que nunca existió, procedió a demolerlo.

Como ya vimos, Rosenstein-Rodan mencionó la necesidad de producir lo que las personas podrían y querrían adquirir como condición para la expansión planeada a gran escala. Le interesaba hallar una solución específica para acelerar la industrialización en un grupo específico de países y bajo circunstancias específicas. La discusión anterior de Nurkse sobre el balance, a la que Hirschman dedicó su atención, ocurrió, como ya se comentó, en un análisis del círculo vicioso de la pobreza, en el que aparecía el "balance"-la necesidad de invertir en la producción de cosas para las que hubiese demanda- junto con la indivisibilidad del capital y la inelasticidad de la demanda, como condiciones limitantes de la tasa de crecimiento y no como explicaciones. La formulación del círculo vicioso o trampa de Nurkse está abierta a la crítica, como he intentado mostrar, pero esta no fue la base de las críticas de Hirschman. La observación casual de Nurkse de que el requisito del equilibrio se podía satisfacer con una mayor 
inversión en un "número de industrias diferentes" y en una "amplia gama de actividades" como sostenía Rosenstein-Rodan, o mediante el tipo de intervención del gobierno japonés o, como evidentemente prefería Nurkse, mediante las oleadas de innovación de Schumpeter, difícilmente parece una base suficiente para decir que la "teoría del crecimiento" de Nurkse es una teoría del "balance" y aún menos para identificarla con el Gran Impulso de Rosenstein-Rodan, como hizo Hirschman.

En el tratamiento de este último, la doctrina del "crecimiento balanceado" se convierte en una forma particular del Gran Impulso en la que "un pueblo" o un gobierno debe erigir "toda una serie de industrias capaces de absorber la producción cada una de las demás". Luego de transformar el Gran Impulso en una teoría del crecimiento balanceado, procedió a criticar el crecimiento balanceado insistiendo en que la versión del Gran Impulso de Rosenstein-Rodan requería capacidades que no posee un país subdesarrollado. "Porque esta es la principal crítica que quiero hacerle a la teoría del crecimiento balanceado: su aplicación requiere precisamente cantidades enormes de aquellas capacidades cuya oferta es muy limitada en los países subdesarrollados" (Hirschman, 1958, p. 53, cursivas mías).

En aras de la claridad, es conveniente separar los diversos elementos que se han agrupado y combinado como doctrina del crecimiento balanceado.

En primer lugar está el diagnóstico de bajo crecimiento, que subraya los impedimentos que surgen de mercados pequeños de lento crecimiento y de "enormes" requerimientos de capital y rápido crecimiento de la población. Esto se refiere a problemas centrados en la falta de incentivos suficientes para invertir, proporciones de factores, criterios de inversión y uso y asignación de recursos. No se puede describir precisamente como una teoría o doctrina de balance. Esto ya se discutió en el capítulo XVII ${ }^{3}$.

En segundo lugar están las repercusiones de una inversión sobre otras o de una demanda sobre otras. Esta es una extensión del concepto de economías de escala externas al aumento en la demanda del producto de un proyecto individual que aparece como resultado del crecimiento general del mercado. Allyn Young ligó el viejo concepto de las ventajas de la división del trabajo al concepto marshalliano de economías internas y externas, y Rosenstein-Rodan y Scitovsky lo extendieron a las economías derivadas de cualquier crecimiento de la demanda, distinguiendo entre economías técnicas y economías

${ }^{3}$ Se refiere al libro inédito de cuyo borrador se toma el presente capítulo. 
pecuniarias externas. Schumpeter subrayó el carácter general de las repercusiones de un tipo de actividad sobre otro. Esta categoría tan general incluye repercusiones que van desde el efecto multiplicador keynesiano de una expansión inicial del gasto mediante inversiones inducidas hasta las "verdaderas" economías de escala resultantes de un menor costo unitario de producción debido al crecimiento del mercado, gama que abarca un gran número de teorías económicas.

En tercer lugar, la palabra "balance" se usa en varios sentidos, desde la asignación de recursos para satisfacer la demanda efectiva que resulta de una distribución de ingresos dada -el sentido general en el que Rosenstein-Rodan y Nurkse usan el término- hasta el sentido estricto de equilibrio perfecto en el que desaparecen las ganancias, que señaló Scitovsky. O se puede hablar de un "balance" entre ahorros de pleno empleo e inversión voluntaria, o del "desbalance" en el que se concibe que un exceso o deficiencia de capacidad tiene un efecto estimulante o inducido sobre otra inversión, de modo que la satisfacción de la demanda es compatible con ciertas pérdidas de corto plazo que aparecen como resultado de un exceso o una insuficiencia (temporales) de demanda corriente. También cabe el sentido popular, donde el término se usa como sinónimo de relaciones fijas entre empleo y producto, o como el "balance" entre agricultura e industria, que desemboca en un "desbalance" si la demanda o el progreso tecnológico cambian en un sector más aprisa que en el otro.

Finalmente, existe la incertidumbre de si el "balance" ocurre por sí mismo y es, por tanto, una teoría del crecimiento en respuesta a las fuerzas del mercado, o si el crecimiento es algo que se debe inducir o estimular y, de ser así, si dicho estímulo consiste en crear y satisfacer demandas o inducir la inversión sin pensar en las demandas, y cuál sería, en cada caso, el papel del Estado en la aceleración del desarrollo de un país subdesarrollado no socialista. Esta discusión toca, a su vez, otro tema relacionado: si el crecimiento rápido debe avanzar por etapas o si requiere simultaneidad de la inversión en varios campos.

Es evidente que el tema se ha vuelto muy amplio y difuso y puede significar cualquier cosa, lo que sugiere que el tema del desarrollo no se puede discutir de manera útil en términos de balance y desbalance. A manera de ejemplo, consideremos de nuevo como trata el tema Hirschman (1958). Aunque se refiere continuamente a la "teoría" o "doctrina" del crecimiento balanceado, dirige sus principales críticas contra el "Gran Impulso", o contra una propuesta de política integral para acelerar el desarrollo. No dice claramente qué causaría el desarrollo en ausencia de intervenciones. Por un lado, habla de "la pobreza e 
ineficiencia universales, de la inmensidad de la tarea y de los círculos viciosos entrelazados" que sugieren la necesidad de medidas enérgicas y compensatorias. Por otro lado, descarta la propuesta del Gran Impulso como "escapista" " derrotista”, pues un país subdesarrollado es, por definición, incapaz de emprender un desarrollo simultáneo en muchos frentes (1958, p. 53), y quienes sugieren tal necesidad descuidan "las abundantes pruebas históricas sobre la penetración gradual de la industria que compite con éxito con los artesanos locales, y de los nuevos productos que primero se importan y más tarde se producen localmente" (ibíd.). Sin embargo, él tampoco confía en estas pruebas históricas, pues defiende una política deliberada de "crecimiento desbalanceado".

Por tanto, nos quedamos sin una teoría o explicación del desarrollo, pero con la necesidad de intervención. Nunca explica satisfactoriamente por qué un país subdesarrollado es capaz de una política deliberada de desarrollo desbalanceado, pero no de un desarrollo balanceado, quizá porque el término desbalance, como lo usa Hirschman, implica producción sin referencia al mercado, con la certeza de sufrir pérdidas y tropezar con cuellos de botella durante un periodo más o menos extenso: desde luego, es más fácil producir con pérdidas que con ganancias. Así, una condición que los autores anteriores trataron como obstáculo para el crecimiento - la posibilidad de pérdidas- se convierte en un beneficio positivo. El apoyo a esta interpretación se basa en su afirmación de que un país subdesarrollado carece de las capacidades para emprender simultáneamente numerosos proyectos (ibíd., p. 55). O bien los proyectos desbalanceados no requieren capacidades, porque se espera que reporten pérdidas y se emprenden en forma gradual y sucesiva. Pero, ¿qué ocurre con los numerosos proyectos que son posibles gracias a los “eslabonamientos"? Cuanto mayor es el número de eslabonamientos, mayores son las capacidades y aptitudes para tomar las decisiones que serán necesarias para obtener los beneficios esperados, las cuales solo aparecerán si dichos eslabonamientos dan lugar a nuevas inversiones.

Hirschman argumenta que "la ventaja del avance intermitente y sorpresivo sobre el 'crecimiento balanceado', en el que toda actividad se expande al ritmo de las demás, es que deja un amplio margen para las decisiones de inversión inducidas y por tanto economiza... la toma de decisiones auténtica" (ibíd., p. 63). Esta es una disyuntiva totalmente falsa. La posibilidad de una expansión en la que toda actividad está perfectamente sincronizada con las demás en un país subdesarrollado (¡o desarrollado!) es ridícula. No es necesario restringir la toma de 
decisiones de inversión "correctas" en los países subdesarrollados con base en la posible desaparición de las ganancias o porque la tendencia a la igualación de las ganancias desaliente nuevas inversiones. Como Adolph Lowe (1965, p. 310) observa con ironía: "Cuando nos enfrentamos al éxito limitado que quizá logren incluso los más denodados esfuerzos por balancear el desarrollo económico en la mayoría de las regiones involucradas, no debemos preocuparnos por un pequeño desequilibrio". En contra de Hirschman, cuanto mayor sea el número de inversiones no rentables menor será el incentivo para hacer nuevas inversiones. Además, no tiene sentido la distinción entre una decisión de inversión "inducida" y una toma de decisiones auténtica.

Esta es, entonces, la respuesta de Hirschman a la posibilidad del "equilibrio del subdesarrollo", o a una tasa de crecimiento demasiado lenta. El problema no existe, puesto que los países desarrollados crecieron mediante una serie de avances industriales graduales, pero, para superar este fantasma, propone romper las barreras convenciendo a los prestamistas internacionales y a los capitalistas y gobiernos locales para que realicen inversiones desbalanceadas ("equivocadas" en términos de la demanda efectiva corriente) que crearán desequilibrio y tensiones, lo que inducirá nuevas inversiones para corregir los errores y, por tanto, jeconomiza toma de decisiones!

Esto se podría denominar estrategia de desarrollo tambaleante, ya que el progreso se asemejaría a los esfuerzos de un borracho por recuperar el equilibrio y seguir adelante mientras se bambolea de un lado a otro. Incluso llega a decir que la presión demográfica provoca contrapresiones y que estas pueden ayudar a la causa del desarrollo, por lo que Hirschman adopta una "visión más calmada" acerca de la explosión demográfica (ibíd., p. 182), que "figura entre los mecanismos de inducción" (ibíd., p. 181).

Por otro lado, la insistencia en la escasez de capacidad para tomar decisiones en los países subdesarrollados sugiere que no está satisfecho con la tasa de crecimiento que consiguen alcanzar con los esfuerzos graduales, puesto que la "escasez" debe estar relacionada con alguna condición.

Es difícil creer que Hirschman realmente se refiera a una escasez de "capacidad para tomar decisiones", aunque usa este término con frecuencia. Lo que seguramente tiene en mente es un incentivo insuficiente para invertir en vez de capacidad para tomar decisiones. Si es lo primero, esto sugeriría que su diagnóstico difiere poco del de Nurkse y de muchos otros autores que han subrayado el punto de los incentivos insuficientes. Su verdadera querella no es con una "teoría 
del desarrollo", sino con una propuesta específica para acelerar el desarrollo que él llama "teoría del crecimiento balanceado".

No confía en las fuerzas del mercado porque piensa que hay "escasez" de "capacidad para tomar decisiones"; cree que la intervención del Estado o de otro tipo para estimular la inversión, la producción y el empleo, si está dirigida a satisfacer la demanda efectiva actual y la recién creada es irrealista e ingenua, pero que la intervención para estimular la inversión que no satisfaga demanda efectiva alguna es realista.

Por razones nunca explicadas, espera que este último tipo de intervención genere "eslabonamientos" (hacia adelante, hacia atrás, verticales, horizontales y laterales) con repercusiones sobre otras empresas y sobre la demanda, y cree así otras oportunidades de inversión (¿balanceadas?), aunque al mismo tiempo diga que las intervenciones para inducir inversiones que satisfagan las demandas carecerían de tales eslabonamientos. En resumen, dice que se aprende cometiendo errores, pero no haciendo lo correcto.

Es interesante señalar el énfasis que Hirschman pone en los eslabonamientos, pues el concepto de eslabonamiento se basa en los esfuerzos o en la respuesta del sistema económico para restablecer el balance o equilibrio creado por una oportunidad de ganancias (desequilibrio o desbalance) o en el deseo de minimizar las pérdidas superando cuellos de botella (desequilibrio).

Después de rechazar la búsqueda de ganancias anticipándose a las demandas efectivas, confía en que funcionará para las inversiones que espera generar con las inversiones equivocadas originales (desbalanceadas), solo porque serán “inducidas" por los eslabonamientos. Cabe preguntar por qué los intentos de anticipar las demandas son irrealistas en un caso pero realistas en el otro, o por qué faltan capacidades para diseñar proyectos que aceleren el desarrollo anticipando las demandas así creadas, pero están ampliamente disponibles para los proyectos que resultan de otros esfuerzos iniciales para aumentar el producto y la demanda. No hay ninguna discusión sobre este punto, que es vital, porque la principal crítica de Hirschman al enfoque del Gran Impulso radica precisamente en la supuesta falta de capacidades adecuadas para iniciarlo y llevarlo a cabo.

Incluso se podría decir irónicamente que el Gran Impulso sería coherente con el argumento de Hirschman porque, como se carece de capacidades para hacer inversiones rentables, también en este caso se incurriría en pérdidas y se crearían "desequilibrios, presiones y tensiones" cuya solución resultaría en un proceso de "aprendizaje". 
Por todo lo anterior, la principal crítica de Hirschman al enfoque del Gran Impulso no es consistente con el enfoque que él propone. Claro que su respuesta sería que no favorece ningún proyecto, salvo aquellos que implican los eslabonamientos máximos, o de máxima repercusión, o en los que un país aprende más. Pero este argumento no le permite eludir la acusación de inconsistencia, pues su selección y su ejecución requieren "capacidad para tomar decisiones", las mismas "capacidades", de las que, según argumenta, se carece para llevar a cabo un desarrollo balanceado.

Hirschman no se contentó con imaginar y luego demoler una teoría del crecimiento balanceado. Procedió a defender una política de crecimiento desbalanceado. Al comienzo de la exposición de su teoría positiva, distingue entre la teoría "pura" del crecimiento balanceado y la menos rigurosa que permite un efecto de "vaivén", un balance solo a largo plazo y un margen considerable para la inversión inducida. Esta se parece a la posición de Nurkse, excepto que Nurkse permitía el desbalance temporal tanto del lado de la oferta como de la demanda, mientras que Hirschman solo contempla desbalances del lado de la oferta.

De inmediato surgen dos preguntas: ¿quién defiende la teoría "pura" del crecimiento balanceado, y en qué se diferencia el crecimiento "desbalanceado" del planteamiento "menos riguroso" de crecimiento balanceado que acabo de citar?

En respuesta a la primera pregunta, Hirschman cita a Scitovsky, quien después de comentar la característica oscilante real del crecimiento, escribió: "el equilibrio solo se logrará cuando las dosis sucesivas de inversión y de expansión en las dos industrias conduzcan a la eliminación simultánea de la inversión en ambas "(citado por Hirschman, 1958, p. 66), y solo cuando no haya más economías pecuniarias externas que impidan que la rentabilidad privada corresponda con la deseabilidad social. Cualquier lector imparcial reconocerá esto como un simple recurso académico que afirma la tendencia del mecanismo de fijación de precios a lograr un objetivo final de equilibrio que nunca se alcanza, y lo relacionará con la preocupación de Scitovsky por la naturaleza de las economías externas. Pero Hirschman elige interpretarlo como la defensa de Scitovsky de una teoría, alegando que propone "cortocircuitos" en el proceso de inversiones para inducir otras inversiones y "alcanzar con un solo salto un nuevo punto de equilibrio donde se ha logrado la "eliminación de la inversión"' (ibíd.). Y continúa, "la política de desarrollo debe ocuparse de erigir la clase de secuencias y repercusiones tan acertadamente descritas por Scitovsky, 
en lugar de intentar suprimirlas" (ibíd., p. 66, cursivas añadidas). Quizá esta grotesca acusación llevó mucho después a Scitovsky (1959) a escribir un artículo que defendía específicamente el rápido crecimiento alcanzable mediante el crecimiento "desbalanceado" (el cual señaló, sin embargo, es compatible con un crecimiento balanceado en otros contextos). En otras palabras, parece muy cuestionable que algún autor defendiera la "teoría pura del crecimiento balanceado".

Nos queda entonces la interesante pregunta de cómo y en qué difiere la "teoría desbalanceada" de la "teoría del balance menos rigurosa”, presumiblemente atribuida a Nurkse. Es una pregunta difícil de responder. Ambas parecen preocupadas por los incentivos para invertir. En opinión de Hirschman "la tarea de la política de desarrollo es mantener las tensiones, desproporciones y desequilibrios" (ibíd., p. 66) mientras que Nurkse ve las "desproporcionalidades" como una consecuencia necesaria de la intervención del gobierno o de las oleadas de innovaciones tipo Schumpeter. Parece ser una cuestión de énfasis. Scitovsky y Nurkse consideraron el deseo de obtener ganancias como incentivo para la inversión. Pero Hirschman no parece disputar esto. Al hablar de "complementariedad" y "necesidad impulsada", señala que existe complementariedad en "toda situación en donde un aumento de la demanda de la mercancía A y el consiguiente aumento de su producción provoque un aumento de la demanda de la mercancía B a su precio corriente"(ibíd., pp. 67-68). Rosenstein-Rodan, Nurkse y Scitovsky podrían haber suscrito esto. De ahí que la afirmación aparentemente paradójica de Hirschman de que "la secuencia que 'nos aleja del equilibrio' es precisamente el patrón ideal de desarrollo" (ibíd., pp. 66-67), parece ser un mero juego de palabras.

Él busca aquí crear un problema afirmando que el significado convencional de "inversión inducida" es que la inversión está directamente relacionada con incrementos anteriores del producto (ibíd., $\mathrm{p}$. 70), mientras que su propio concepto de inversión inducida se limita a las inversiones que se espera que se beneficien más de las economías externas de lo que se beneficiarán otras industrias creando economías externas, es decir, "deben ser beneficiarias netas de economías externas" (ibíd., p. 71). Abandona prontamente este razonamiento tortuoso al afirmar que "seguiremos diciendo que la inversión induce otras inversiones y lo único que haremos es tener conciencia de que hay diversos grados de 'inducciones"'(ibíd.). Al salir de ese embrollo, olvidó señalar que también abandonaba el intento de distinguir su tratamiento de los incentivos del tratamiento propuesto por los autores que criticaba. 
Otro intento de distinguir entre su posición de crecimiento "desbalanceado" y la posición de crecimiento balanceado "menos rigurosa" se basa en su concepto de la principal restricción del desarrollo: la falta de "capacidad para tomar decisiones". Pero si esto tiene algún sentido, seguramente es una forma curiosa de referirse a la falta de incentivos suficientes para invertir. Quien haya trabajado en países subdesarrollados dará fe de que no faltan proyectos y propuestas grandiosos, es decir, no hay falta de capacidad para "tomar decisiones", sino falta de capacidad para tomar "buenas" decisiones; es decir, buenas desde el punto de vista del desarrollo, y también dará fe de que faltan incentivos adecuados para invertir porque el entorno es desfavorable, porque el tamaño mínimo de ciertas inversiones tropieza con la estrechez del mercado o porque existe escasez de divisas, etc.

Pero es posible que Hirschman realmente quiera decir lo que dice: falta de capacidad para tomar decisiones, ya que en un trabajo posterior (Hirschman, 1967) parece acercarse peligrosamente a recomendar el ocultamiento de riesgos y circunstancias negativas, exagerando en cambio los beneficios esperados para inducir ciertas decisiones. Esta pieza de economía dudosa y moralidad aún más dudosa se exalta en "el Principio de la Mano Escondida": "una manera de inducir la acción mediante el error, donde el error es una subestimación de los costos o dificultades del proyecto" (ibíd., p. 29).

Se podría pensar que, debido a su énfasis en la complementariedad, en los eslabonamientos y en la inversión inducida, era de esperar que Hirschman defendiera el enfoque del Gran Impulso para acelerar el desarrollo. Su principal razón para no defenderlo -la supuesta falta de capacidad de los países subdesarrollados para planear y la falta de las capacidades requeridas- nada tiene que ver con el crecimiento balanceado o desbalanceado y está igualmente presente en el tipo de proyectos que él defiende. Seguramente no puede rechazar el enfoque debido al peligro de que sea tan exitoso que resulte en un equilibrio perfecto y no haya más incentivos para invertir. Puesto que piensa que los países subdesarrollados son totalmente incapaces de un gran esfuerzo más o menos organizado, lógicamente debería defender las “tensiones, desproporciones y desequilibrios" que crearía ese Gran Impulso y el valioso aprendizaje que nacería de los esfuerzos por corregir los desequilibrios resultantes.

La conclusión que parece surgir de este examen es que no existe el dilema entre una teoría del crecimiento balanceado y otra del crecimiento desbalanceado, y que se dilapidaron mucho tiempo y 
muchos esfuerzos valiosos al abordarla. Peor aún, desvió la atención de los problemas reales y apremiantes que, por ello, se descuidaron.

E1 principal problema que plantearon Rosenstein-Rodan, Scitovsky y Nurkse, y más tarde Nelson, Leibenstein y Myrdal, fue el de la trampa o círculo vicioso del subdesarrollo o, en forma más simple y general, la incapacidad de las fuerzas del mercado para enfrentar adecuadamente los factores depresores. Diferentes autores subrayaron, en diferente grado, la naturaleza y la fuerza de los factores depresores y, por tanto, las soluciones que cada uno consideraba más adecuadas, pero todos los autores mencionados coincidían en el punto central de que era necesaria una acción consciente y deliberada para salir de la trampa y acelerar el desarrollo.

Incluso Hirschman reconoció un impedimento específico y propuso medidas para aliviarlo. Pero su identificación del Gran Impulso con el desarrollo balanceado y la condena general de ambos influyeron, sin duda, en que ignorara o menospreciara el problema principal que preocupaba a los autores que criticó.

Más tarde, la identificación del crecimiento con un aumento positivo del PIB per cápita y la demostración estadística de que casi todos los países subdesarrollados estaban experimentando dicho aumento, y posiblemente la sustitución de los términos países "en desarrollo" o "menos desarrollados" por "subdesarrollados", todo eso conspiró para crear la sensación de que no existe una trampa del subdesarrollo, que el crecimiento avanza lenta pero seguramente, y que, por tanto, no se requieren un Gran Impulso ni medidas heroicas de algún tipo. Pero esa es otra historia.

Aquí falta mencionar las propuestas positivas de Hirschman: que todos los fenómenos económicos están relacionados y que toda variación de precio, innovación, inversión, cambio en la demanda y en la oferta de factores tienen repercusiones de largo alcance sobre otros fenómenos económicos, observación que constituye el núcleo de la teoría del equilibrio, de la teoría de formación de precios y del proceso de crecimiento.

Pero difícilmente se puede hacer una teoría útil a partir de los desequilibrios y defender el desequilibrio como una política consciente. Lo que se puede hacer es sopesar la fuerza relativa de los factores que deprimen y estimulan el ingreso, las fuerzas que impulsan la innovación, el progreso técnico y la inversión con respecto a las fuerzas que llevan a continuar la economía dual, donde un sector importante de la economía avanza poco o nada, y promover medidas que fortalezcan a las primeras y debiliten a las segundas. 
La idea de que los países subdesarrollados y las agencias de crédito deberían concentrar sus esfuerzos en proyectos específicos, seleccionados para crear deliberadamente tensiones y desproporciones, e intentar comparar unos con otros calculando el grado y la naturaleza de las posibles repercusiones o eslabonamientos, parece ser completamente impráctica. Por ejemplo, ¿cuál es el resultado neto probable de las repercusiones creadas al sobrestimar o subestimar deliberadamente la demanda de energía eléctrica en comparación con tratar de estimar la demanda probable y construirla?

Como después señaló Michael Lipton (1962), Hirschman termina con un modelo terriblemente complicado, con distinciones entre gastos sociales generales y actividades directamente productivas, actividad forzada e inducida, actividad estatal y privada, $y$ actividades de bienes básicos y de bienes de consumo. Los proyectos no solo se deben clasificar en estas categorías, sino que se desconocen los eslabonamientos posteriores. Además, aunque el enfoque es complicado omite muchas de las fuerzas más importantes que estimulan y deprimen el ingreso.

La secuencia que aleja del equilibrio como patrón ideal y la defensa del crecimiento desbalanceado pueden ser ideas intrigantes para algunos autores académicos, pero difícilmente proporcionan criterios factibles de inversión para lograr mejores proporciones entre factores.

Es interesante observar el breve debate de Rosenstein-Rodan (1961) sobre la teoría o, más propiamente hablando, sobre la política del Gran Impulso, incluido en un trabajo presentado en la reunión de la Asociación Económica Internacional de 1957 en Río de Janeiro, pero que solo se publicó en 1961. Se titulaba "Notas sobre la teoría del Gran Impulso” y era una exposición breve y muy condensada.

No había leído a Hirschman, pero pienso que por influencia de Nurkse señaló que "la teoría del crecimiento es en gran medida una teoría de la inversión” (1961, p. 57). Con el paso del tiempo y la aparición de nuevas condiciones, el tratamiento muestra algunos cambios interesantes. Las modificaciones más significativas parecen ser el mayor énfasis en las indivisibilidades de la oferta, en especial de infraestructura social, y en las economías externas. Se mantiene el argumento básico de que una serie de inversiones simultáneas -el Gran Impulso- creará la demanda requerida de su producto. Pero la versión de 1961 relaciona el desarrollo con un aumento de los incentivos para invertir más que con el empleo y el producto, y la necesidad y la indivisibilidad de la infraestructura social aparecen 
como el primer y mayor obstáculo que se debe superar, el cual, en términos de inversión, en una sociedad cerrada, quizá ascienda a tres cuartas partes del total ${ }^{4}$.

Explica la falta de crecimiento de los países subdesarrollados durante el siglo XIX como consecuencia de deficiencias en su infraestructura social. El comercio internacional redujo el tamaño del quantum mínimo de inversión, pero no evita la necesidad de un Gran Impulso. Agrega un interesante punto adicional sobre el impacto psicológico de la velocidad y el tamaño del desarrollo y sobre la probabilidad de que una cantidad de esfuerzos pequeños y aislados no tenga suficiente impacto sobre el crecimiento (ibíd., p. 66). No menciona el "balance", salvo por implicación cuando se refiere al hecho de que "las bajas elasticidades de la demanda hacen mucho más difícil ajustar las ofertas a la demanda” (ibíd., p. 63), lo que ya había señalado Nurkse.

Nurkse comentó el artículo de Rosenstein-Rodan. El cambio de énfasis de este último hacia la infraestructura social parecía totalmente aceptable para Nurkse. Afirmó que, para él, "el punto sustantivo más importante, subrayado en el documento que tenemos ante nosotros, es que la inversión pública general crea oportunidades de inversión en actividades directamente productivas" y que "el capital general se debe construir antes que la demanda” (Nurkse, 1961, p. 75). De modo que el autor calificado como principal exponente de una "teoría del crecimiento balanceado" no encontró dificultad o incoherencia en abogar por la creación, en ciertas circunstancias, de una capacidad para producir bienes y servicios antes de que exista la respectiva demanda.

De nuevo, no se mencionó el balance. Pero, aunque el cambio de énfasis de Rosenstein-Rodan hacia la infraestructura social hacía más aceptable la teoría del Gran Impulso, también disminuyó la fuerza y el impacto de la presentación anterior. Nurkse preguntó por qué el calificativo "Gran Impulso"?

¿Hay una teoría rival del Pequeño Impulso? Casi todos coincidimos en la importancia de las inversiones públicas generales, en la complementariedad entre las necesidades de los consumidores, en la necesidad de grandes ahorros y en el papel vital de la empresa, privada y pública, es decir, en el impulso al desarrollo (ibíd., p. 74).

${ }^{4}$ Ibíd., p. 60. Fue este énfasis en la infraestructura social lo que me llevó a atemperar mi reconocimiento a la contribución de Rosenstein-Rodan y a omitir la estrecha relación entre el Gran Impulso y la idea del Rompimiento que defendí en Desarrollo económico acelerado (Currie, 1966, pp. 74-75). 
Aunque después aceptó que "depender únicamente del sistema de precios para los cambios estructurales que constituyen el desarrollo puede ser insuficiente" (ibíd., p. 75), la cuestión básica de la necesidad o conveniencia de un esfuerzo crítico mínimo se desdibujó y se perdió de vista. El desempleo disfrazado o la mano de obra sumamente subutilizada quedaron fuera de escena.

El crecimiento balanceado se introdujo en la discusión con el resumen de Alexandre Kafka, quien atribuyó a Nurkse la idea de que "el principio del crecimiento balanceado no le parecía ser un componente necesario de la teoría del Gran Impulso" (1961, p. 78). En general, la discusión fue muy insatisfactoria y no se centró en lo que debería haber sido el tema clave: si era necesario o deseable un Gran Impulso o un esfuerzo crítico mínimo para acelerar el desarro1lo. Mostró, sin embargo, que, aún en 1957, ni Rosenstein-Rodan ni Nurkse pensaban que existía un problema real entre el crecimiento balanceado y el desbalanceado, ni identificaron la propuesta de acelerar el desarrollo mediante un esfuerzo masivo con "la teoría del crecimiento balanceado".

$\mathrm{Si}$ "balance" se entiende en el sentido de la motivación y el impulso básicos del sistema de libre empresa, constituye una "teoría" del crecimiento defendible en la explicación de la secuencia histórica seguida por la mayoría de los países hoy desarrollados. Pero esta es solo otra manera de decir que, en ciertos entornos favorables, el sistema tiene en sí mismo un impulso suficiente hacia la innovación y la reducción de costos. Difícilmente parece apropiado o descriptivo caracterizarla como "la teoría del crecimiento balanceado". Los autores que dirigían sus críticas al crecimiento balanceado apuntaban al objetivo equivocado.

Una parte esencial del argumento del presente escrito es que los cambios de circunstancia han empeorado las posibilidades de que el sistema de libre empresa pueda funcionar en los países subdesarrollados tal como lo hizo en los países desarrollados, excepto en los casos cada vez más raros donde el entorno es favorable. Por tanto, lo que se debe examinar no son tanto las fuerzas básicas del cambio económico como las condiciones y circunstancias cambiantes en las que operan. La disyuntiva balance-desbalance fue una pista falsa que solo sirvió para desviar la atención de problemas más importantes.

Es tentador evitar la discusión del crecimiento balanceadodesbalanceado como si solo tuviera interés para el historiador del pensamiento económico. La razón para insistir en ella es el impacto desafortunado que tuvo en las propuestas para un ataque masivo al 
problema del subdesarrollo. E1 "Gran Impulso" pareció anticipar este campo y, a su vez, se llegó a identificar con el crecimiento balanceado y se convirtió en el blanco de muchas críticas dirigidas contra este concepto.

Hasta donde sé, ningún autor intentó separar los temas o reformular la propuesta de Rosenstein-Rodan para satisfacer las críticas válidas. El mismo no intentó llevarla más allá de la etapa de idea general, y afirmó que "aquí se confirma la atención a lo que se debe hacer y no a cómo se debe hacer". Ni siquiera defendió la idea durante catorce años (dieciocho años si nos atenemos la fecha de las publicaciones) y después se conformó con un programa de obras públicas de infraestructura que todos los gobiernos y organismos internacionales estaban impulsando. Nurkse siguió convencido de que el mecanismo de precios, sin otra ayuda, sería insuficiente para lograr un crecimiento rápido, pero se conformó con un programa ecléctico y difuso. Por alguna razón inexplicable, el magistral argumento de Leibenstein (1957) sobre la necesidad de un esfuerzo crítico mínimo no se identificó con la necesidad de un Gran Impulso y no se expuso en términos específicos para países específicos. Mi propio intento (Currie, 1966) sufrió, sin duda, por su presentación algo apresurada y poco convencional.

No obstante, la necesidad de un Gran Impulso o esfuerzo crítico mínimo es cada día más urgente en la mayoría de los países subdesarrollados. Los programas de ayuda convencionales y el crecimiento continuo que registran las engañosas cifras del PIB per cápita solo sirven para ocultar el alarmante deterioro del entorno demográfico y político, la brecha creciente de los niveles de consumo y el empobrecimiento que deterioran, día tras día, la efectividad del sistema de precios.

Los recursos domésticos subutilizados y mal utilizados, la deficiente movilidad, los inadecuados y mal utilizados recursos de intercambio, las indivisibilidades técnicas, la inelasticidad de la demanda, los sistemas de precios y de competencia que funcionan mal, el empeoramiento de la capacidad para competir en el comercio internacional, la población en rápido crecimiento y los entornos políticos y sociales desfavorables: todos ellos, en sus diversos grados y combinaciones, deben tenerse en cuenta para que sea efectivo un nuevo ataque al problema del subdesarrollo. Si no, la tarea puede pasar por defecto a sistemas económicos mejor equipados para movilizar las economías $\mathrm{y}$ conseguir un rompimiento en este frente. 


\section{REFERENCIAS BIBLIOGRÁFICAS}

Currie, L. (1966). Accelerating development: The necessity and the means. Nueva York: McGraw-Hill.

Fleming, M. (1955). External economies and the doctrine of balanced growth. Economic Journal, 65(258), 241-256.

Hirschman, A. O. (1958). The strategy of economic development. New Haven: Yale University Press.

Hirschman, A. O. (1963). Development projects observed. Washington DC: The Brookings Institution.

Kafka, A. (1961). The theoretical interpretation of Latin American economic development. En H. S. Ellis y H. C. Wallich (eds.). Economic development of Latin America (pp. 1-28). Londres: Macmillan.

Leibenstein, H. (1957). Economic backwardness and economic growth. Nueva York: Wiley.

Lowe, A. (1965). On economic knowledge. Nueva York: Harper \& Rowe.

Nurkse, R. (1953). Problems of capital formation in underdeveloped countries. Oxford: Basil Blackwell.

Nurkse, R. (1957). The conflict between 'balanced growth' and international specialization and some reflections on the international financing of public overhead investments. Lectures on Economic Development. Istanbul University. Reimpresión: "Balanced and unbalanced growth", en Haberler, G. y Stern, R. M. (eds.). (1961). Equilibrium and growth in the world economy: Economic essays by Ragnar Nurkse (pp. 241-278). Cambridge, Mass.: Harvard University Press.

Rosenstein-R., P. (1943). Problems of industrialisation of Eeastern and South-Eastern Europe. Economic Journal, 53(210-211), 202-211.

Rosenstein-R., P. (1961). Notes on the theory of the 'big push'. En H. S. Ellis y H. C. Wallich (eds.). Economic Development of Latin America (pp. 57-81). Londres: Macmillan.

Scitovsky, T. (1954). Two concepts of external economies. Journal of Political Economy, 62(2), 143-151.

Scitovsky, T. (1959). Growth balanced or unbalanced? En Abramovitz, M. et al. (eds.). The allocation of economic resources: Essays in honor of Bernard Francis Haley (pp. 201-217). Stanford, Ca.: Stanford University Press.

Young, A. A. (1928). Increasing returns and economic progress. Economic Journal, 38(152), 527-542. Trad. al español: Young, A. A. (2009). Rendimientos crecientes y progreso económico, Revista de Economía Institucional, 11(21), 227-243. 